\title{
Elastostatic Deformation Analysis of Thick Isotropic Beams by Using Different Beam Theories and a Meshless Method
}

\author{
Armagan Karamanli* \\ *Department of Mechatronics Engineering, Faculty of Engineering and Architecture, Istanbul Gelisim University, 34215 \\ Istanbul, Turkey. \\ (afkaramanli@gelisim.edu.tr) \\ *Corresponding Author; Armagan Karamanli, Department of Mechatronics Engineering, Faculty of Engineering and \\ Architecture, Istanbul Gelisim University, 34215 Istanbul, Turkey, Tel: +90 2124227020, armagan_k@yahoo.com
}

Received: 17.05.2016 Accepted: 27.07.2016

\begin{abstract}
The elastostatic deformations of thick isotropic beams subjected to various sets of boundary conditions are presented by using different beam theories and the Symmetric Smoothed Particle Hydrodynamics (SSPH) method. The analysis is based on the Euler-Bernoulli, Timoshenko and Reddy-Bickford beam theories. The performance of the SSPH method is investigated for the comparison of the different beam theories for the first time. For the numerical results, various numbers of nodes are used in the problem domain. Regarding to the computed results for RBT, various number of terms in the Taylor Series Expansions (TSEs) is employed. To validate the performance of the SSPH method, comparison studies in terms of transverse deflections are carried out with the analytical solutions. It is found that the SSPH method has provided satisfactory convergence rate and smaller L2 error.
\end{abstract}

Keywords Meshless Method, Element-Free, Beam, Euler-Bernoulli, Timoshenko, Reddy-Bickford.

\section{Introduction}

The kinematics of deformation of a beam can be represented by using various beam theories. Among them, the Euler Bernoulli Beam Theory (EBT), the Timoshenko Beam Theory (TBT) and the Reddy-Bickford Beam Theory (RBT) are commonly used. The effect of the transverse shear deformation neglected in the EBT is allowed in the latter two beam theories.

Euler Bernoulli Beam Theory is the simplest beam theory and assumes that the cross sections which are normal to the mid-plane before deformation remain plane/straight and normal to the mid-plane after deformation. Both transverse shear and transverse normal strains are neglected by using these assumptions. In the TBT, the normality assumption of the EBT is relaxed and the cross sections do not need to normal to the mid-plane but still remain plane. The TBT requires the shear correction factor (SCF) to compensate the error due to the assumption of the constant transverse shear strain and shear stress through the beam thickness. The SCF depends on the geometric and material parameters of the beam but the loading and boundary conditions are also important to determine the SCF [1-2]. In the third order shear deformation theory which is named as the RBT, the transverse shear strain is quadratic trough the thickness of the beam [3].

The need for the further extension of the EBT is raised for the engineering applications of the beam problems often characterized by high ratios, up to 40 for the composite structures, between the Young modulus and the shear modulus [4]. Various higher order beam theories are introduced in which the straightness assumption is removed and the vanishing of shear stress at the upper and lower surfaces are accommodated. For this purpose, higher order polynomials incorporating either one, or more, extra terms [5-11] or trigonometric functions [12-13] or exponential functions [14] are included in the expansion of the longitudinal point-wise displacement component through the thickness of the beam. The higher order theories introduce additional unknowns that make the governing equations 
more complicated and provide the solutions much costly in terms of CPU time. The theories which are higher than the third order shear deformation beam theory are seldom used because the accuracy gained by these theories which require much effort to solve the governing equations is so little [4].

The beam theories are still the reference technique in many engineering applications. They continue to be advantageous in the analysis of slender bodies such as airplane wings, helicopter blades, bridges and frames where the cumbersome two-dimensional 2D (plate and shell theories) and three-dimensional 3D analysis require higher cost and computational effort because of their complexity.

Meshless methods are widely used in static and dynamic analyses of the engineering beam problems [15-20]. To obtain the approximate solution of the problem by a meshless method, the selection of the basis functions is almost the most important issue. The accuracy of the computed solution can be increased by employing different number of terms in TSE or increasing number of nodes in the problem domain or by increasing the degree of complete polynomials. Many meshless methods have been proposed by researchers to obtain the approximate solution of the problem. The Smoothed Particle Hydrodynamics (SPH) method is proposed by Lucy [21] to the testing of the fission hypothesis. However, this method has two important shortcomings, lack of accuracy on the boundaries and the tensile instability. To remove these shortcomings, many meshless methods have been proposed such as the Corrected Smoothed Particle Method [22,23], Reproducing Kernel Particle Method [24-26], Modified Smoothed Particle Hydrodynamics (MSPH) method [27-30], the Symmetric Smoothed Particle Hydrodynamics method [31-36] and the Strong Form Meshless Implementation of Taylor Series Method [37-38], Moving Kringing Interpolation Method [3940], the meshless Shepard and Least Squares (MSLS) Method [42], Spectral Meshless Radial Point Interpolation (SMRPI) Method [42].

It is seen form the above literature survey regarding to the SSPH method, there is no reported work on the elastostatic deformations of the thick isotropic beams subjected to the different boundary conditions by employing the TBT and RBT.

Linear elastic problems including quasi-static crack propagation [31-33], crack propagation in an adhesively bonded joint [34], 2D Heat Transfer problems [35] and 1D 4th order nonhomogeneous variable coefficient boundary value problems [36] have been successfully solved by employing the SSPH method.

The SSPH method has an advantage over the MLS, RKPM, MSPH and the SMITSM methods because basis functions used to approximate the function and its derivatives are derived simultaneously and even a constant weight function can be employed to obtain the approximate solution [31-36]. The matrix to be inverted for finding kernel estimates of the trial solution and its derivatives is asymmetric in the MSPH. In SSPH method which made the matrix to be inverted symmetric, reduced the storage requirement and the CPU time.
In view of the above, the objectives of this paper mainly are to present the SSPH method formulation for the isotropic thick beams subjected to different boundary conditions within the framework of EBT, TBT and RBT, to perform numerical calculations to obtain the transverse deflections of the studied beam problems and finally to compare the results obtained by using the SSPH method with analytical solutions. It is believed that researchers will probably find the SSPH method helpful to solve their engineering problems.

In section 2, the formulation of the EBT, TBT and RBT is. In section 3, the formulation of the SSPH method is given for 1D problem. In Section 4, numerical results are given based on the two types of engineering beam problem which are a simply supported beam under uniformly distributed load and a cantilever beam under the uniformly distributed load. The performance of the SSPH method is compared with the analytical solutions.

\section{Formulation of Beam Theories}

To describe the EBT, TBT and RBT, the following coordinate system is introduced. The $\mathrm{X}$-coordinate is taken along the axis of the beam and the z-coordinate is taken through the height (thickness) of the beam. In the general beam theory, all the loads and the displacements $(\mathrm{u}, \mathrm{w})$ along the coordinates $(\mathrm{x}, \mathrm{z})$ are only the functions of the $\mathrm{x}$ and $\mathrm{z}$ coordinates. [4] The formulation of the EBT, TBT and RBT are given below.

\subsection{Euler Bernoulli Beam Theory}

The following displacement field is given for the EBT,

$u(x, z)=-z \frac{d w}{d x}$

where $\mathrm{w}_{0}$ is the transverse deflection of the point $(\mathrm{x}, 0)$ which is on the mid-plane $(\mathrm{z}=0)$ of the beam. By using the assumption of the smallness of strains and rotations, the only the axial strain which is nonzero is given by,

$\varepsilon_{x x}=\frac{d u}{d x}=-z \frac{d^{2} w_{0}}{d x^{2}}$

The virtual strain energy of the beam in terms of the axial stress and the axial strain can be expressed by

$\delta U=\int_{0}^{L} \int_{A} \sigma_{x x} \delta \varepsilon_{x x} d A d x$

where $\delta$ is the variational operator, $\mathrm{A}$ is the cross sectional area, $\mathrm{L}$ is the length of the beam, $\sigma_{x x}$ is the axial stress. The bending moment of the EBT is given by,

$M_{x x}=\int_{A} z \sigma_{x x} d A$

By using equation (2) and equation (4), equation (3) can be rewritten as,

$\delta U=-\int_{0}^{L} M_{x x} z \frac{d^{2} \delta w_{0}}{d x^{2}}$ 
The virtual potential energy of the load $q(x)$ which acts at the central axis of the beam is given by

$\delta V=-\int_{0}^{L} q(x) \delta w_{0} d x$

If a body is in equilibrium, $\delta \mathrm{W}=\delta \mathrm{U}+\delta \mathrm{V}$, the total virtual work $(\delta \mathrm{W})$ done equals zero. Then one can obtain,

$\delta W=-\int_{0}^{L}\left(M_{x x} z \frac{d^{2} \delta w_{0}}{d x^{2}}+q(x) \delta w_{0}\right) d x=0$

After performing integration for the first term in equation (7) twice and since $\delta w_{0}$ is arbitrary in $(0<\mathrm{x}<\mathrm{L})$, one can obtain the following equilibrium equation,

$-\frac{d^{2} M_{x x}}{d x^{2}}=q(x)$ for $0<x<L$

By introducing the shear force $Q_{x}$ and rewrite equation (8) in the following form

$-\frac{d M_{x x}}{d x}+Q_{x}=0, \quad-\frac{d Q_{x}}{d x}=q(x)$

By using Hooke's law, one can obtain

$\sigma_{x x}=E \varepsilon_{x x}=-E z \frac{d^{2} w_{0}}{d x^{2}}$

where $\mathrm{E}$ is the modulus of elasticity. If the equation (10) is put into equation (4), it is obtained,

$M_{x x}=-\int_{A} E z^{2} \frac{d^{2} w_{0}}{d x^{2}} d A=-D_{x} \frac{d^{2} w_{0}}{d x^{2}}$

where $D_{x x}=E I_{y}$ is the flexural rigidity of the beam and $I_{y}=\int_{A} z^{2} d A$ the second moment of area about the y-axis. The substitution of equation (11) into equation (9) yields the EBT governing equation

$\frac{d^{2}}{d x^{2}}\left(D_{x x} \frac{d^{2} w_{0}}{d x^{2}}\right)=q(x)$ for $0<x<L$

\subsection{Timoshenko Beam Theory}

The following displacement field is given for the TBT,

$u(x, z)=z \phi(x)$

$w(x, z)=w_{0}(x)$

where $\phi(x)$ is the rotation of the cross section. By using equation (13), the strain-displacement relations are given by

$\varepsilon_{x x}=\frac{d u}{d x}=-z \frac{d \phi}{d x}$

$\gamma_{x z}=\frac{d u}{d z}+\frac{d w}{d x}=\phi+\frac{d w_{0}}{d x}$

The virtual strain energy of the beam including the virtual energy associated with the shearing strain can be written as,

$\delta U=\int_{0}^{L} \int_{A}\left(\sigma_{x x} \delta \varepsilon_{x x}+\sigma_{x z} \delta \gamma_{x z}\right) d A d x$

where $\sigma_{x Z}$ is the transverse shear stress and $\gamma_{x Z}$ is the shear strain. The bending moment and the shear force can be written respectively,

$$
M_{x x}=\int_{A} z \sigma_{x x} d A, \quad Q_{x}=\int_{A} \sigma_{x z} d A
$$

By using equation (14) and equation (16), one can rewrite equation (15) as,

$\delta U=\int_{0}^{L}\left[M_{x x} \frac{d \delta \phi}{d x}+Q_{x}\left(\delta \phi+\frac{d \delta w_{0}}{d x}\right)\right] d x$

The virtual potential energy of the load $q(x)$ which acts at the central axis of the Timoshenko beam is given by

$\delta V=-\int_{0}^{L} q(x) \delta w_{0} d x$

Since the total virtual work done equals zero and the coefficients of $\delta \phi$ and $\delta w_{0}$ in $0<\mathrm{x}<\mathrm{L}$ are zero, one can obtain the following equations,

$-\frac{d M_{x x}}{d x}+Q_{x}=0, \quad-\frac{d Q_{x}}{d x}=q(x)$

The bending moment and shear force can be expressed in terms of generalized displacement $\left(w_{0}, \phi\right)$ by using the constitutive equations $\sigma_{x x}=E \varepsilon_{x x}$ and $\sigma_{x z}=G \gamma_{x z}$,

$M_{x x}=\int_{A} z \sigma_{x x} d A=D_{x} \frac{d \phi}{d x}$

$Q_{x}=\kappa_{s} \int_{A} \sigma_{x Z} d A=\kappa_{s} A_{x Z}\left(\phi+\frac{d w_{0}}{d x}\right)$

Where $\kappa_{S}$ is the shear correction factor, $\mathrm{G}$ is the shear modulus, $D_{x x}=E I_{y}$ is the flexural rigidity of the beam and $A_{x z}=G A$ is the shear rigidity. The SCF is used to compensate the error caused by the assumption of a constant transverse shear stress distribution along the beam thickness. The governing equations of the TBT is obtained in terms of generalized displacements by substituting equation (20) and equation (21) into equation (19),

$-\frac{d}{d x}\left(D_{x x} \frac{d \phi}{d x}\right)+\kappa_{s} A_{x z}\left(\phi+\frac{d w_{0}}{d x}\right)=0$

$-\frac{d}{d x}\left[\kappa_{s} A_{x z}\left(\phi+\frac{d w_{0}}{d x}\right)\right]=q(x)$

\subsection{Reddy-Bickford Beam Theory}

The following displacement field is given for the RBT,

$u(x, z)=z \phi(x)-\alpha z^{3}\left(\phi(x)+\frac{d w(x)}{d x}\right)$

$w(x, z)=w_{0}(x)$

where $\alpha=4 /\left(3 h^{2}\right)$. By using equation (24), the straindisplacement relations of the RBT are given by

$\varepsilon_{x x}=\frac{d u}{d x}=z \frac{d \phi}{d x}-\alpha z^{3}\left(\frac{d \phi}{d x}+\frac{d^{2} w_{0}}{d x^{2}}\right)$

$\gamma_{x z}=\frac{d u}{d z}+\frac{d w}{d x}=\phi+\frac{d w_{0}}{d x}-\beta z^{2}\left(\phi+\frac{d w_{0}}{d x}\right)$

where $\beta=3 \alpha=4 /\left(h^{2}\right)$.

The virtual strain energy of the beam can be written as,

$\delta U=\int_{0}^{L} \int_{A}\left(\sigma_{x x} \delta \varepsilon_{x x}+\sigma_{x z} \delta \gamma_{x z}\right) d A d x$

The usual bending moment and the shear force are,

$M_{x x}=\int_{A} z \sigma_{x x} d A, \quad Q_{x}=\int_{A} \sigma_{x z} d A$

and $P_{x x}$ and $R_{x}$ are the higher order stress resultants can be written respectively, 


$$
P_{x x}=\int_{A} z^{3} \sigma_{x x} d A, \quad R_{x}=\int_{A} z^{2} \sigma_{x z} d A
$$

By using equation (25), equation (27) and equation (2.28), one can rewrite the equation (26) as,

$\delta U=\int_{0}^{L}\left[\left(M_{x x}-\alpha P_{x x}\right) \frac{d \delta \phi}{d x}-\alpha P_{x x} \frac{d^{2} \delta w_{0}}{d x^{2}}+\left(Q_{x}-\right.\right.$ $\left.\left.\beta R_{x}\right)\left(\delta \phi+\frac{d \delta w_{0}}{d x}\right)\right] d x$

In the RBT there is no need to use a SCF unlike the TBT. The virtual potential energy of the transverse load $q(x)$ is given by

$\delta V=-\int_{0}^{L} q(x) \delta w_{0} d x$

The virtual displacements principle is applied and the coefficients of $\delta \phi$ and $\delta w_{0}$ in $0<\mathrm{x}<\mathrm{L}$ are set to zero, the governing equations of the RBT are obtained in terms of displacements $\phi$ and $w_{0}$ as follows,

$-\frac{d}{d x}\left(\bar{D}_{x x} \frac{d \phi}{d x}-\alpha \hat{F}_{x x} \frac{d^{2} w_{0}}{d x^{2}}\right)+\bar{A}_{x z}\left(\phi+\frac{d w_{0}}{d x}\right)=0$

$-\alpha \frac{d^{2}}{d x^{2}}\left(\widehat{F}_{x x} \frac{d \phi}{d x}-\alpha H_{x x} \frac{d^{2} w_{0}}{d x^{2}}\right)-\frac{d}{d x}\left[\bar{A}_{x z}\left(\phi+\frac{d w_{0}}{d x}\right)\right]=q(x)$

where

$\bar{A}_{x z}=\hat{A}_{x z}-\beta \widehat{D}_{x z}, \quad \bar{D}_{x x}=\widehat{D}_{x x}-\alpha \widehat{F}_{x x}$

$\widehat{D}_{x x}=D_{x x}-\alpha F_{x x}, \quad \hat{F}_{x x}=F_{x x}-\alpha H_{x x}$

$\hat{A}_{x z}=A_{x z}-\beta D_{x z}, \widehat{D}_{x z}=D_{x z}-\beta F_{x z}$

$\left(D_{x x}, F_{x x}, H_{x x}\right)=\int_{A}\left(z^{2}, z^{4}, z^{6}\right) E d A$

$\left(A_{x z}, D_{x z}, F_{x z}\right)=\int_{A}\left(1, z^{2}, z^{4}\right) G d A$

\section{Formulation of Symmetric Smoothed Particle Hydrodynamics}

Taylor Series Expansion (TSE) of a scalar function can be given by

$f\left(\xi_{1}\right)=\sum_{m=0}^{n} \frac{1}{m !}\left[\left(\xi_{1}-x_{1}\right) \frac{d}{d x_{1}}\right]^{m} f\left(x_{1}\right)$

where $f\left(\xi_{1}\right)$ is the value of the function at $\xi=\left(\xi_{1}\right)$ located in near of $\mathrm{x}=\left(\mathrm{x}_{1}\right)$. If the zeroth to sixth order terms are employed and the higher order terms are neglected, the equation (33) can be written as follows,

$f(\xi)=P(\xi, x) Q(x)$

where

$Q(x)=\left[f(x), \frac{d f(x)}{d x_{1}}, \frac{1}{2 !} \frac{d^{2} f(x)}{d x_{1}{ }^{2}}, \ldots, \frac{1}{6 !} \frac{d^{6} f(x)}{d x_{1}{ }^{6}}\right]^{T}$

$P(\xi, x)=\left[1,\left(\xi_{1}-x_{1}\right),\left(\xi_{1}-x_{1}\right)^{2}, \ldots,\left(\xi_{1}-x_{1}\right)^{6}\right]$

To determine the unknown variables given in the $\mathrm{Q}(\mathrm{x})$, both sides of equation (34) are multiplied with $\mathrm{W}(\xi, \mathrm{x}) \mathrm{P}(\xi, \mathrm{x})^{\mathrm{T}}$ and evaluated for every node in the CSD. The following equation is obtained where $\mathrm{N}(\mathrm{x})$ is the number nodes in the compact support domain (CSD) of the $\mathrm{W}(\xi, \mathrm{x})$ as shown in Fig. 1.

$$
\begin{aligned}
& \sum_{j=1}^{N(x)} f\left(\xi^{r(j)}\right) W\left(\xi^{r(j)}, x\right) P\left(\xi^{r(j)}, x\right)^{T} \\
& =\sum_{j=1}^{N(x)}\left[P\left(\xi^{r(j)}, x\right)^{T} W\left(\xi^{r(j)}, x\right) P\left(\xi^{r(j)}, x\right)\right] Q(x)
\end{aligned}
$$

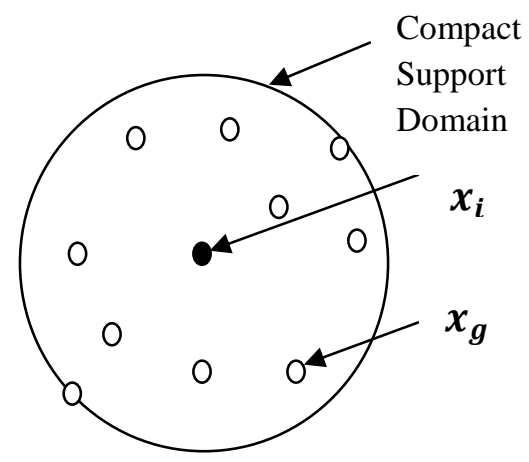

Fig. 1. Compact support of the weight function $W(\xi, x)$ for the node located at $\mathrm{x}=\left(\mathrm{x}_{\mathrm{i}}, \mathrm{y}_{\mathrm{i}}\right)$

Then, equation (37) can be given by

$C(\xi, x) Q(x)=D(\xi, x) F^{(x)}(\xi, x)$

where $\quad \mathrm{C}(\xi, \mathrm{x})=\mathrm{P}(\xi, \mathrm{x})^{\mathrm{T}} \mathrm{W}(\xi, \mathrm{x}) \mathrm{P}(\xi, \mathrm{x})$ and $\mathrm{D}(\xi, \mathrm{x})=$ $\mathrm{P}(\xi, \mathrm{x})^{\mathrm{T}} \mathrm{W}(\xi, \mathrm{x})$.

The solution of equation (38) is given by

$Q(x)=K(\xi, x) F(\xi)$

where $\mathrm{K}^{(\mathrm{x})}(\xi, \mathrm{x})=\mathrm{C}(\xi, \mathrm{x})^{-1} \mathrm{D}(\xi, \mathrm{x})$. Equation (39) can be also written as follows

$Q_{I}(x)=\sum_{J=1}^{M} K_{I J} F_{J}, \quad I=1,2, \ldots, 6$

Where $M$ is the number of nodes and $F_{J}=f\left(\xi^{J}\right)$. Seven components of equation (40) for 1D case are can be written as

$$
\begin{aligned}
& f(x)=Q_{1}(x)=\sum_{J=1}^{M} K_{1 J} F_{J} \\
& \frac{d f(x)}{d x_{1}}=Q_{2}(x)=\sum_{J=1}^{M} K_{2 J} F_{J} \\
& \frac{d^{2} f(x)}{d x_{1}^{2}}=2 ! Q_{3}(x)=\sum_{J=1}^{M} K_{3 J} F_{J} \\
& \frac{d^{3} f(x)}{d x_{1}^{3}}=3 ! Q_{4}(x)=\sum_{J=1}^{M} K_{4 J} F_{J} \\
& \frac{d^{4} f(x)}{d x_{1}^{4}}=4 ! Q_{5}(x)=\sum_{J=1}^{M} K_{5 J} F_{J} \\
& \frac{d^{5} f(x)}{d x_{1}^{5}}=5 ! Q_{6}(x)=\sum_{J=1}^{M} K_{6 J} F_{J} \\
& \frac{d^{6} f(x)}{d x_{1}^{6}}=6 ! Q_{7}(x)=\sum_{J=1}^{M} K_{7 J} F_{J}
\end{aligned}
$$

\section{Numerical Results}

The pure bending of two engineering beam problems by using the formulation of the EBT, TBT and RBT are solved by using the SSPH method. Different loading and boundary conditions are applied with different node distributions in the problem domain. For the numerical solutions obtained by the RBT are evaluated with different node distributions in the 
problem domain and varying number of terms in the TSEs. The numerical results obtained by the SSPH method regarding to different beam theories are compared with the analytical solution of problem.

\subsection{Simply Supported Beam}

Static transverse deflections of a simply supported beam under uniformly distributed load of intensity $q_{0}$ as shown in Fig.2. is studied.

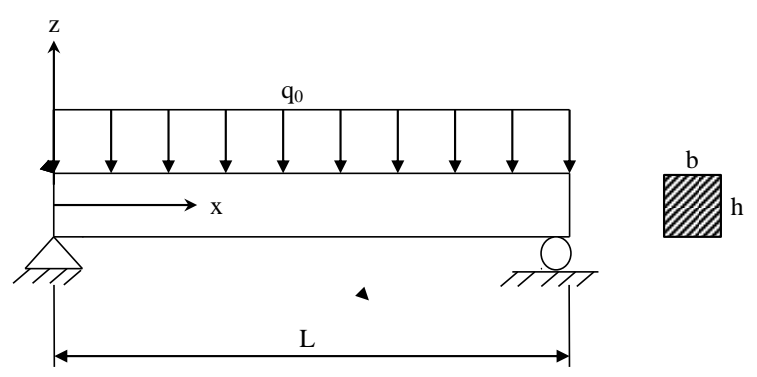

Fig. 2. Simply supported beam with uniformly distributed load

The physical parameters of the beam are given as $\mathrm{L}=2 \mathrm{~m}$, $\mathrm{h}=0.2 \mathrm{~m}, \mathrm{~b}=0.02 \mathrm{~m}$. Modulus of elasticity $\mathrm{E}$ is $210 \mathrm{GPa}$, shear modulus $\mathrm{G}$ is $80.8 \mathrm{GPa}$ and the distributed load q_0 is set to $150000 \mathrm{~N} / \mathrm{m}$.

Based on the EBT, the governing equation of the problem can be given by,

$\frac{d^{2}}{d x^{2}}\left(D_{x x} \frac{d^{2} w_{0}}{d x^{2}}\right)=q_{0} \quad$ for $0<x<L$

where $D_{x x}=E I_{y}$ is the flexural rigidity of the beam and $I_{y}=b h^{3} / 12$ the second moment of area about the y-axis. The boundary conditions regarding to the EBT are given as follows

$x=0, \quad \frac{d^{2} w_{0}}{d x^{2}}=0$ and $w_{0}=0 \mathrm{~m}$

$x=L, \quad \frac{d^{2} w_{0}}{d x^{2}}=0$ and $w_{0}=0 \mathrm{~m}$

The analytical solution of this boundary value problem based on the EBT is given by

$w_{0}^{E}(x)=\frac{q_{0} L^{4}}{24 D_{x x}}\left(\frac{x}{L}-\frac{2 x^{3}}{L^{3}}+\frac{x^{4}}{L^{4}}\right)$

where the superscript $E$ denotes the quantities in the EBT.

The governing equations of the problem can be written by using TBT as follows,

$-\frac{d}{d x}\left(D_{x x} \frac{d \phi}{d x}\right)+\kappa_{s} A_{x z}\left(\phi+\frac{d w_{0}}{d x}\right)=0$

$-\frac{d}{d x}\left[\kappa_{s} A_{x z}\left(\phi+\frac{d w_{0}}{d x}\right)\right]=q_{0}$

where $D_{x x}=E I_{y}$ is the flexural rigidity of the beam, $I_{y}=b h^{3} / 12$ is the second moment of area about the $y$ axis, $A_{x z}=G A=G b h$ is the shear rigidity and the SCF is assumed to be constant $\kappa_{s}=5 / 6$ for the rectangular cross section.
The boundary conditions regarding to the TBT are given as follows;

$$
\begin{gathered}
x=0, \quad \frac{d \phi}{d x}=0 \text { and } w_{0}=0 \mathrm{~m} \\
x=L, \quad \frac{d \phi}{d x}=0 \text { and } w_{0}=0 \mathrm{~m}
\end{gathered}
$$

The analytical solution of this boundary value problem based on the TBT is given by

$w_{0}{ }^{T}(x)=\frac{q_{0} L^{4}}{24 D_{x x}}\left(\frac{x}{L}-\frac{2 x^{3}}{L^{3}}+\frac{x^{4}}{L^{4}}\right)+\frac{q_{0} L^{2}}{2 \kappa_{S} A_{x z}}\left(\frac{x}{L}-\frac{x^{2}}{L^{2}}\right)$

where the superscript $T$ denotes the quantities in the TBT.

The governing equations of the problem can be written by using RBT as follows,

$-\frac{d}{d x}\left(\bar{D}_{x x} \frac{d \phi}{d x}-\alpha \hat{F}_{x x} \frac{d^{2} w_{0}}{d x^{2}}\right)+\bar{A}_{x z}\left(\phi+\frac{d w_{0}}{d x}\right)=0$

$-\alpha \frac{d^{2}}{d x^{2}}\left(\hat{F}_{x x} \frac{d \phi}{d x}-\alpha H_{x x} \frac{d^{2} w_{0}}{d x^{2}}\right)-\frac{d}{d x}\left[\bar{A}_{x Z}\left(\phi+\frac{d w_{0}}{d x}\right)\right]=q$

where $D_{x x}=E I_{y}$ is the flexural rigidity of the beam, $I_{y}=b h^{3} / 12$ is the second moment o farea about the $\mathrm{y}$ axis, $A_{x z}=G A=G b h$ is the shear rigidity, $\alpha=4 /\left(3 h^{2}\right)$ and $\beta=4 /\left(h^{2}\right) . \bar{D}_{x x}, \bar{A}_{x z}, \hat{F}_{x x}, H_{x x}$ are calculated according to the equations given in equation (32).

The boundary conditions regarding to the TBT are given as follows

$x=0, \widehat{D}_{x x} \frac{d \phi}{d x}-\alpha F_{x x} \frac{d^{2} w_{0}}{d x^{2}}=0$, and $w_{0}=0 \mathrm{~m}$

$x=L, \quad \widehat{D}_{x x} \frac{d \phi}{d x}-\alpha F_{x x} \frac{d^{2} w_{0}}{d x^{2}}=0$, and $\quad w_{0}=0 \mathrm{~m}$

The analytical solution of this boundary value problem based on the RBT is given by

$$
\begin{gathered}
w_{0}^{T}(x)=\frac{q_{0} L^{4}}{24 D_{x x}}\left(\frac{x}{L}-\frac{2 x^{3}}{L^{3}}+\frac{x^{4}}{L^{4}}\right)+\left(\frac{q_{0} \mu}{\lambda^{4}}\right)\left(\frac{\widehat{D}_{x x}}{\hat{A}_{x z} D_{x x}}\right) \\
{\left[-\tanh \left(\frac{\lambda L}{2}\right) \sinh \lambda x+\cosh \lambda x+\frac{\lambda^{2}}{2} x(L-x)-1\right]}
\end{gathered}
$$

where

$\lambda^{2}=\frac{\bar{A}_{x z} D_{x x}}{\alpha\left(F_{x x} \widehat{D}_{x x}-\hat{F}_{x x} D_{x x}\right)}, \quad \mu=\frac{\hat{A}_{x z} \widehat{D}_{x z}}{\alpha\left(F_{x x} \widehat{D}_{x x}-\hat{F}_{x x} D_{x x}\right)}$

The above boundary value problems are solved by using the SSPH method for the node distributions of 21, 41 and 161 equally spaced nodes in the domain $x \in[0,2]$. As the weight function, the Revised Super Gauss Function (RSGF) which gives the least $\mathrm{L}_{2}$ error norms in numerical solutions in [31] is used.

$$
\begin{gathered}
W(x, \xi)=\frac{G}{(h \sqrt{\pi})^{\lambda}}\left\{\begin{array}{cc}
\left(36-d^{2}\right) e^{-d^{2}} & 0 \leq d \leq 6 \\
0 & d>6
\end{array}\right\} \\
d=|x-\xi| / h
\end{gathered}
$$

where $d$ is the radius of the CSD, $h$ is the smoothing length. $\mathrm{G}$ and $\lambda$ are the parameters which are eliminated by the formulation of the SSPH method.

The numerical solutions are performed according to the following meshless parameters; the radius of the support 
domain (d) is chosen as 6 and the smoothing length (h) equals to $1.1 \Delta$ where $\Delta$ is the minimum distance between two adjacent nodes. The meshless parameters, $\mathrm{d}$ and $\mathrm{h}$, are selected to obtain the lowest error.

For the numerical solutions based on the formulation of the RBT, it is also investigated the effect of the different numbers of terms employed in the TSE when the number of nodes in the problem domain increases. Computed results obtained by using the SSPH method are compared with the analytical solutions, and their accuracy and convergence properties are investigated by employing the global $\mathrm{L}_{2}$ error norm which is given in equation (51).

$$
\| \text { Error } \|_{2}=\frac{\left[\sum_{j=1}^{m}\left(v_{\text {num }}^{j}-v_{\text {exact }}^{j}\right)^{2}\right]^{1 / 2}}{\left[\sum_{j=1}^{m}\left(v_{\text {exact }}^{j}\right)^{2}\right]^{1 / 2}}
$$

The $\mathrm{L}_{2}$ error norms of the numerical solutions based on the EBT are given in Table 1. For the numerical analysis different numbers of nodes are considered in the problem domain with 5 terms in TSEs expansion. It is observed in Table 1 that the accuracy of the SSPH method is not improved by increasing of the number of nodes in the problem domain. At least for the problem studied here, it is impossible to evaluate the convergence rate of the SSPH method because of the level of the numerical errors which are too small obtained for different number of nodes in the problem domain.

It is observed in Fig. 3 that the SSPH method agrees very well with the analytical solution. The transverse deflection of the beam computed by the SSPH method is virtually indistinguishable from that for the analytical solution.

Table 1. $\mathrm{L}_{2}$ error norm for different number of nodes based on EBT

\begin{tabular}{|c|c|c|c|}
\hline \multirow{2}{*}{$\begin{array}{c}\text { Meshless } \\
\text { Method }\end{array}$} & \multicolumn{3}{|c|}{ Number of Nodes } \\
\cline { 2 - 4 } & 21 Nodes & 41 Nodes & 161 Nodes \\
\hline SSPH & $3.8563 \times 10^{-9}$ & $9.0440 \times 10^{-8}$ & $3.6898 \times 10^{-7}$ \\
\hline
\end{tabular}

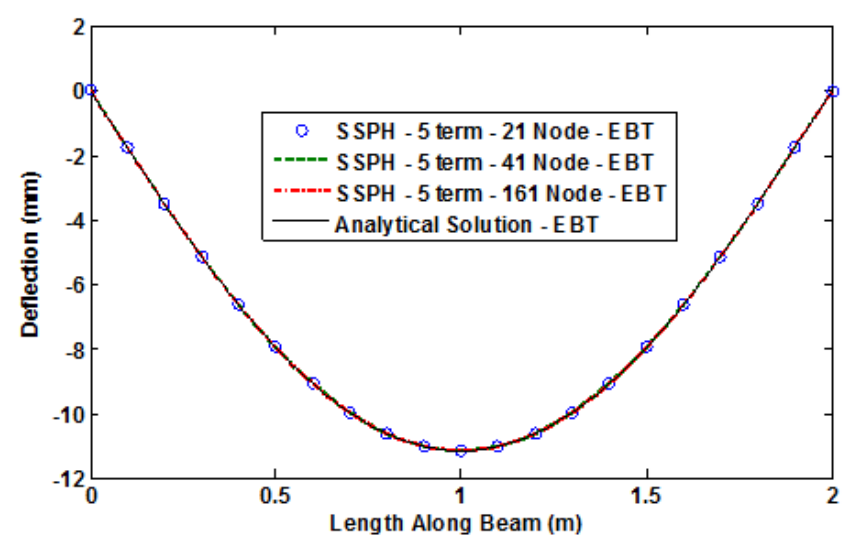

Fig. 3. Deflections of the beam computed based on the EBT and the analytical solution

The global $\mathrm{L}_{2}$ error norms of the solutions based on the TBT are given in Table 2 where different numbers of nodes are considered with 5 terms in TSEs expansion. The results in Table 2 are obtained for the meshless parameters $\mathrm{d}$ and $\mathrm{h}$ which give the best accuracy for each method. It is observed in Table 2 that the SSPH method almost gives the exact solution of the problem. The SSPH method gives accurate values of the displacement even for 21 nodes in the problem domain. It is observed in Fig. 4 that the SSPH method agrees very well with the analytical solution.

Table 2. $\mathrm{L}_{2}$ error norm for different number of nodes based on TBT

\begin{tabular}{|c|c|c|c|}
\hline Meshless & \multicolumn{3}{|c|}{ Number of Nodes } \\
\cline { 2 - 4 } Method & 21 Nodes & 41 Nodes & 161 Nodes \\
\hline SSPH & $4.3044 \times 10^{-10}$ & $3.7090 \times 10^{-9}$ & $3.5981 \times 10^{-9}$ \\
\hline
\end{tabular}

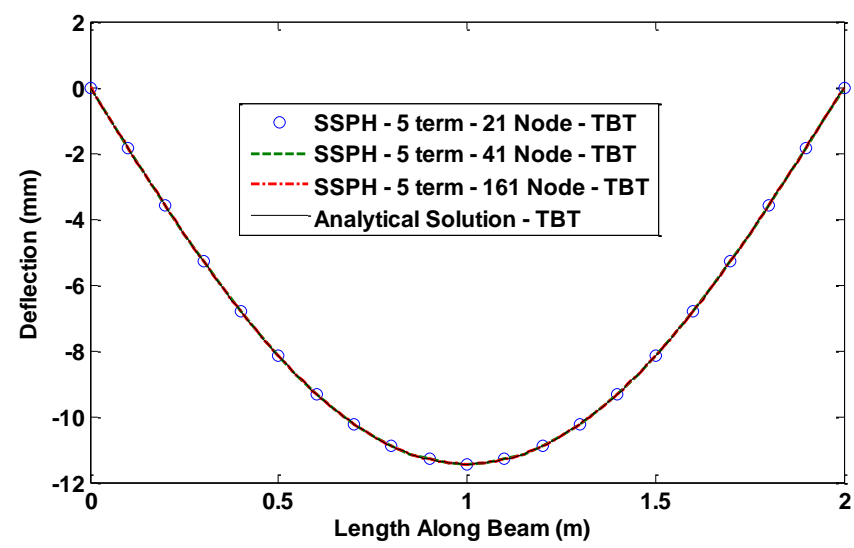

Fig. 4. Deflections of the beam computed based on the TBT and the analytical solution

The global $\mathrm{L}_{2}$ error norms of the solutions based on the RBT are given in Table 3 where different numbers of nodes are considered with varying number of terms in TSEs expansion. The results in Table 3 are obtained for the meshless parameters $\mathrm{d}$ and $\mathrm{h}$ which gives the best accuracy for each method. Different numbers of terms in TSEs, 5 to 7 , are employed to evaluate the performance of the SSPH method. It is found that the convergence rate of the computed solution increases by increasing the degree of complete polynomials. The rate of convergence for the SSPH method increases by increasing the number of nodes in the problem domain. It is clear that numerical solutions obtained by the SSPH method agree very well with the analytical solution given in Fig. 5 to Fig. 7.

Table 3. $\mathrm{L}_{2}$ error norm for different number of nodes with varying number of terms in the TSEs

\begin{tabular}{|c|c|c|c|}
\hline \multirow{2}{*}{ Nodes } & \multicolumn{3}{|c|}{ Terms in the TSEs } \\
\cline { 2 - 4 } & 5 Term & 6 Term & 7 Term \\
\hline 21 & 2.0631 & 2.0475 & 2.0014 \\
\hline 41 & 2.0631 & 2.0317 & 1.6977 \\
\hline 161 & 2.0631 & 1.9371 & 0.5556 \\
\hline
\end{tabular}

Comparison of the analytical solutions in terms of transverse deflections obtained by the EBT, TBT and RBT are given in Fig. 8. It is observed that the analytical solution obtained by the EBT is similar to the analytical solution obtained by the RBT than the TBT. It is clear that the RBT is a higher order shear deformation theory that yields more accurate results than the other theories which are studied in this paper. 


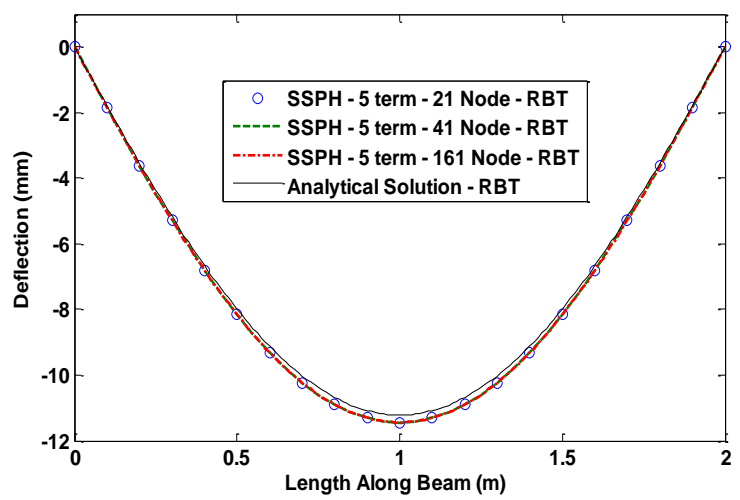

Fig. 5. Deflections of the beam computed based on the RBT and the analytical solution -5 term

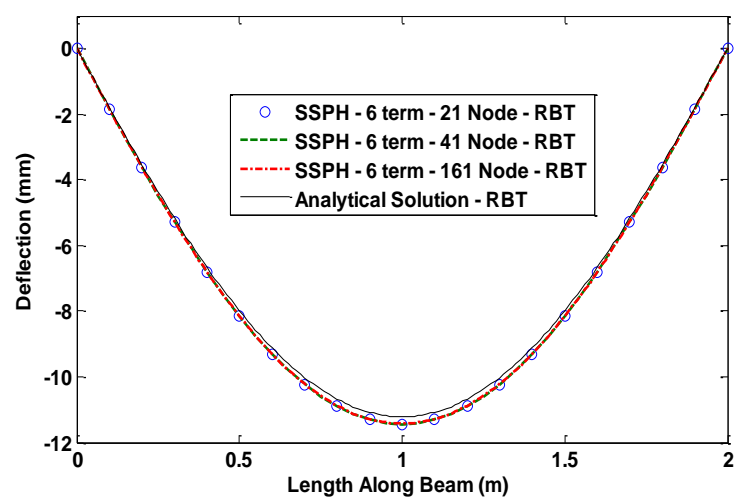

Fig. 6. Deflections of the beam computed based on the RBT and the analytical solution -6 term

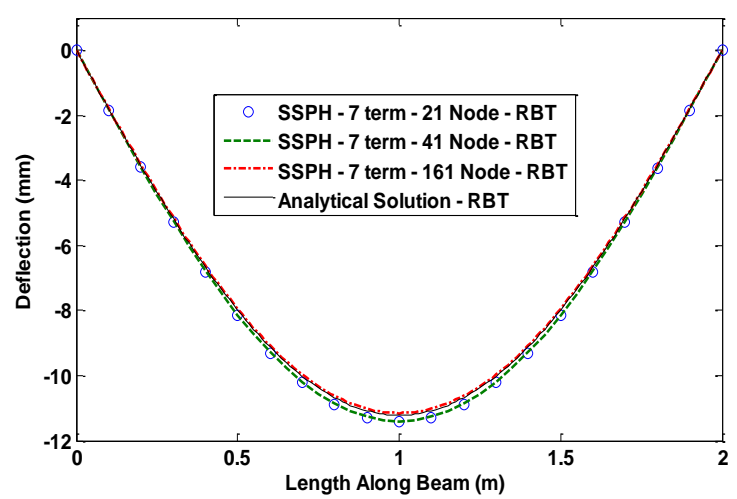

Fig. 7. Deflections of the beam computed based on the RBT and the analytical solution -7 term

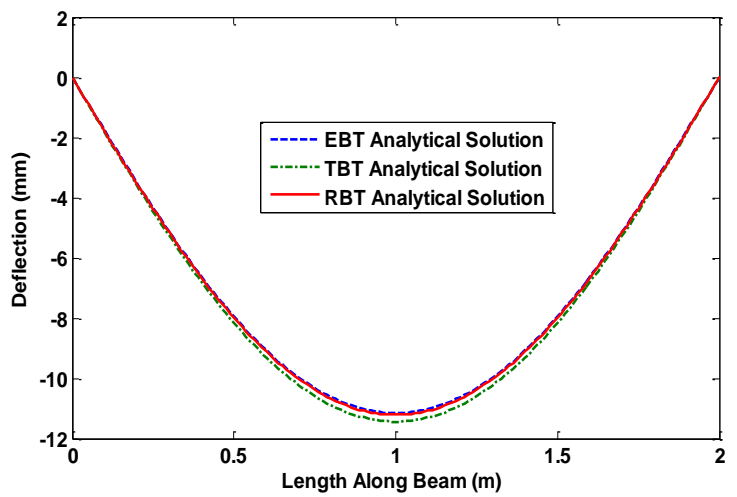

Fig. 8. Comparison of the analytical solutions in terms of deflections obtained by the EBT, TBT and RBT

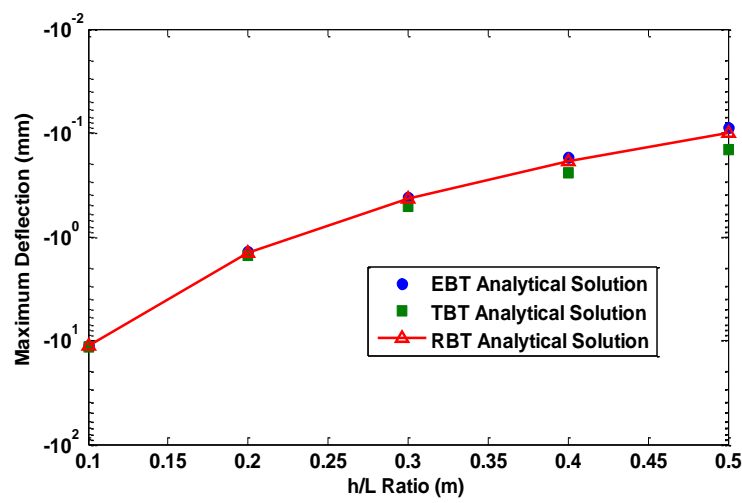

Fig. 9. Comparison of the analytical solutions in terms of maximum deflections obtained with varying $\mathrm{h} / \mathrm{L}$ ratio

For the future studies, the effect of the $\mathrm{h} / \mathrm{L}$ ratio can be investigated to evaluate the accuracy of the TBT in terms of transverse deflection. In Fig. 8, the $\mathrm{h} / \mathrm{L}$ ratio is 0.1 . It is observed in Fig. 9 that when the $\mathrm{h} / \mathrm{L}$ ratio increases the accuracy of the TBT decreases in terms of transverse deflection.

\subsection{Cantilever Beam}

For a cantilever beam the static transverse deflections under uniformly distributed load of intensity $q_{0}$ as shown in Figure 10 is studied.
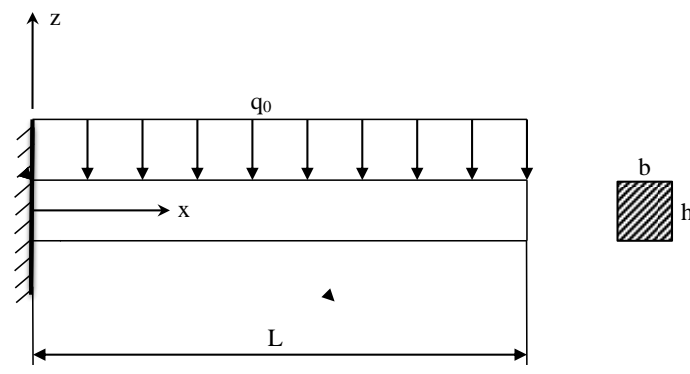

Fig. 10. Simply supported beam with uniformly distributed load

The physical parameters are given as $\mathrm{L}=2 \mathrm{~m}, \mathrm{~h}=0.2 \mathrm{~m}$, $\mathrm{b}=0.02 \mathrm{~m}$. Modulus of elasticity $\mathrm{E}$ is $210 \mathrm{GPa}$, shear modulus $\mathrm{G}$ is $80.8 \mathrm{GPa}$ and the uniformly distributed load $q_{0}$ is set to $50000 \mathrm{~N} / \mathrm{m}$.

Based on the EBT, the governing equation of the problem is as given in equation (42). The boundary conditions are given by;

$x=0, \quad \frac{d w_{0}}{d x}=0$ and $w_{0}=0 \mathrm{~m}$

$x=L, \quad \frac{d^{2} w_{0}}{d x^{2}}=0$ and $\frac{d^{3} w_{0}}{d x^{3}}=0$

The analytical solution of this boundary value problem based on the EBT is given by

$w_{0}^{E}(x)=\frac{q_{0} L^{4}}{24 D_{x x}}\left(6 \frac{x^{2}}{L^{2}}-4 \frac{x^{3}}{L^{3}}+\frac{x^{4}}{L^{4}}\right)$

Based on the TBT, the governing equations of the problem are given in equation (44) and equation (45). The 
boundary conditions regarding to the TBT are given as follows

$x=0, \quad \phi=0$ and $w_{0}=0 \mathrm{~m}$

$x=L, \quad \frac{d \phi}{d x}=0$ and $\phi+\frac{d w_{0}}{d x}=0$

The analytical solution of this boundary value problem based on the TBT is given by

$w_{0}{ }^{T}(x)=\frac{q_{0} L^{4}}{24 D_{x x}}\left(6 \frac{x^{2}}{L^{2}}-4 \frac{x^{3}}{L^{3}}+\frac{x^{4}}{L^{4}}\right)+\frac{q_{0} L^{2}}{2 \kappa_{S} A_{x z}}\left(2 \frac{x}{L}-\frac{x^{2}}{L^{2}}\right)$

Based on the RBT, the governing equations of the problem are given in equation (47) and equation (48). The boundary conditions regarding to the RBT are given as follows

$x=0, \phi=0$ and $w_{0}=0 \mathrm{~m}$

$x=L, \quad \widehat{D}_{x x} \frac{d \phi}{d x}-\alpha F_{x x} \frac{d^{2} w_{0}}{d x^{2}}=0$, and $\phi+\frac{d w_{0}}{d x}=0$

The analytical solution of this boundary value problem based on the TBT is given by

$w_{0}{ }^{R}(x)=w_{0}^{E}(x)+\left(\frac{q_{0} \mu}{2 \lambda^{2}}\right)\left(\frac{\widehat{D}_{x x}}{\widehat{A}_{x z} D_{x x}}\right)\left(2 L x-x^{2}\right)+$ $\left(\frac{q_{0} \mu}{\lambda^{4} \cosh \lambda L}\right)\left(\frac{\widehat{D}_{x x}}{\hat{A}_{x z} D_{x x}}\right)[\cosh \lambda x+\lambda L \sinh \lambda(L-x)-$ $\left.\left(\frac{q_{0} \mu}{\lambda^{4}}\right)\left(\frac{\widehat{D}_{x x}}{\widehat{A}_{x z} D_{x x}}\right)\left(\frac{1+\lambda L \sinh \lambda L}{\cosh \lambda L}\right)\right]$

The above boundary value problems are solved by using the SSPH method for different node distributions of 21,41 and 161 equally spaced nodes in the domain $x \in[0,2]$. The Revised Super Gauss Function given in equation (50) is used as the weight function.

For the numerical solutions, the radius of the support domain (d) is chosen as 5 and the smoothing length (h) is chosen as $1.3 \Delta$. Also, for the numerical solutions based on the RBT, it is investigated the effect of the various numbers of terms employed in the TSEs when the number of nodes in the problem domain increases. The meshless parameters, $d$ and $h$, are selected to obtain the best accuracy. Computed results by the SSPH method are compared with the analytical solutions, and their rate of convergence and accuracy properties are investigated by using the global L2 error norm given in equation (51). In Table 4 the global $\mathrm{L}_{2}$ error norms of the solutions based on the EBT are given for different numbers of nodes in the problem domain with 5 terms in TSEs expansion. The similar case observed in the previous problem is also found in this problem.

The accuracy of the SSPH method is not improved by increasing of the number of nodes in the problem domain. At least for the problem studied here, it is impossible to evaluate the convergence of the SSPH method because of the level of the numerical errors which are too small obtained for different number of nodes in the problem domain. The computed transverse deflection of the beam is virtually indistinguishable from that for the analytical solution as seen from Fig. 12.
Table 4. $\mathrm{L}_{2}$ error norm for different number of nodes based on EBT

\begin{tabular}{|c|c|c|c|}
\hline Meshless & \multicolumn{3}{|c|}{ Number of Nodes } \\
\cline { 2 - 4 } Method & 21 Nodes & 41 Nodes & 161 Nodes \\
\hline SSPH & $9.3439 \times 10^{-8}$ & $5.7719 \times 10^{-6}$ & $7.8041 \times 10^{-6}$ \\
\hline
\end{tabular}

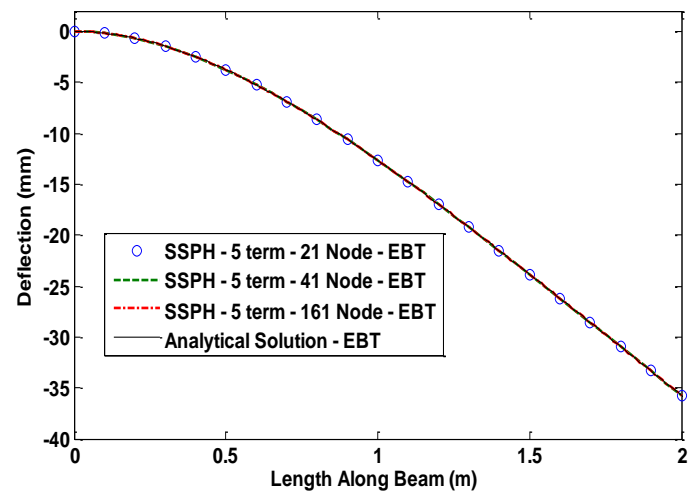

Fig. 12. Deflections of the beam computed based on the EBT and the analytical solution

By using different numbers of nodes in the problem domain with 5 terms in TSEs expansion, the global $\mathrm{L}_{2}$ error norms of the solutions obtained for the TBT are given in Table 5. It is clear in Table 5 that the SSPH method provides satisfactory numerical results and rate of convergence. It is observed in Fig. 13 that the SSPH method agrees very well with the analytical solution.

Table 5. Global $\mathrm{L}_{2}$ error norm for different number of nodes based on TBT

\begin{tabular}{|c|c|c|c|}
\hline \multirow{2}{*}{$\begin{array}{c}\text { Meshless } \\
\text { Method }\end{array}$} & \multicolumn{3}{|c|}{ Number of Nodes } \\
\cline { 2 - 4 } & 21 Nodes & 41 Nodes & 161 Nodes \\
\hline SSPH & $1.1353 \times 10^{-8}$ & $3.2478 \times 10^{-7}$ & $7.2764 \times 10^{-8}$ \\
\hline
\end{tabular}

The global $\mathrm{L}_{2}$ error norms of the solutions based on the RBT are given in Table 6 where different numbers of nodes are considered with varying number of terms in TSEs expansion. It is observed that the convergence rate of the computed solution increases by increasing the degree of complete polynomials for 161 nodes in the problem domain.

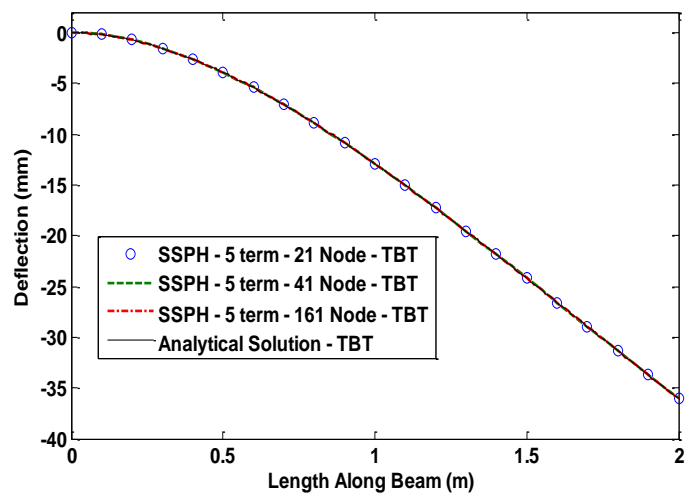

Fig. 13. Deflections of the beam computed based on the TBT and the analytical solution. 
Table 6. $\mathrm{L}_{2}$ error norm for different number of nodes with varying number of terms in the TSEs

\begin{tabular}{|c|c|c|c|}
\hline \multirow{2}{*}{$\begin{array}{c}\text { Number of } \\
\text { Nodes }\end{array}$} & \multicolumn{3}{|c|}{ Number of Terms in the TSEs } \\
\cline { 2 - 4 } & 5 Term & 6 Term & 7 Term \\
\hline 21 & 1.7608 & 1.7608 & 1.7479 \\
\hline 41 & 1.7783 & 1.7784 & 1.8504 \\
\hline 161 & 1.7920 & 1.7919 & 1.5278 \\
\hline
\end{tabular}

The convergence rate of the SSPH method is increasing as the number of nodes is increased in the problem domain even by using same number of terms in the TSEs. It is clear that the transverse displacement computed with the SSPH method closer to the analytical solution of the problem given in Fig. 14 to Fig. 16.

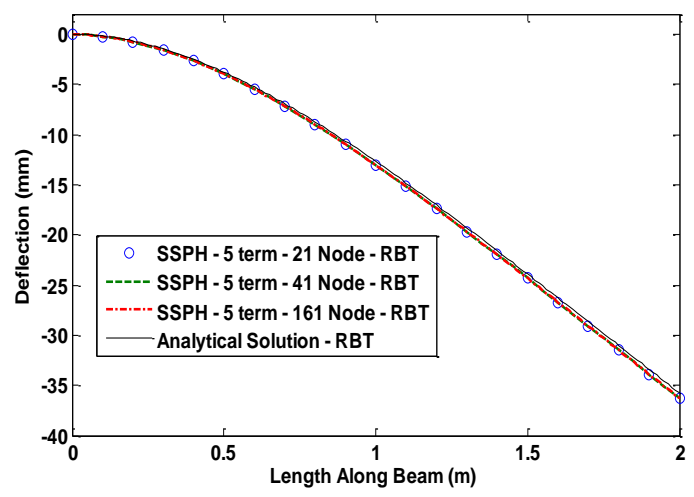

Fig. 14. Deflections of the beam based on the RBT along the $\mathrm{X}$-axis computed by the SSPH method using different number of nodes and the analytical solution -5 term

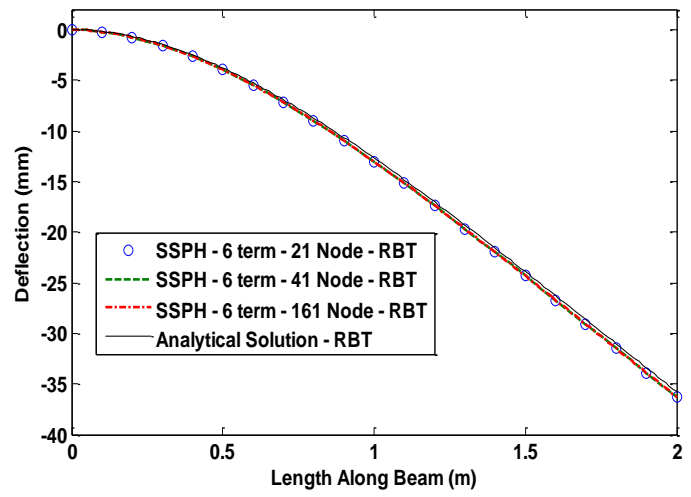

Fig. 15. Deflections of the beam based on the RBT along the $\mathrm{x}$-axis computed by the SSPH method using different number of nodes and the analytical solution -6 term

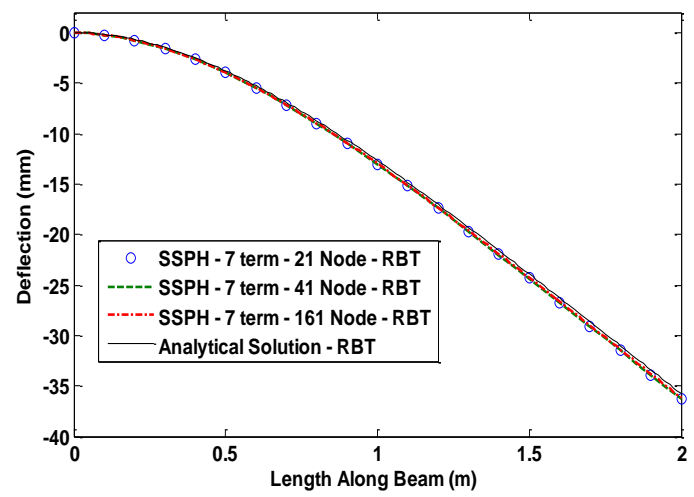

Fig. 16. Deflections of the beam based on the RBT along the $\mathrm{X}$-axis computed by the SSPH method using different number of nodes and the analytical solution -7 term

The analytical solutions of the EBT, TBT and RBT are compared in Fig. 17. It is clear that the analytical solution obtained by the EBT is more close to the analytical solution obtained by the RBT than the TBT. At least for the problem studied here, the EBT yields more accurate results in terms of transverse deflection than the TBT.

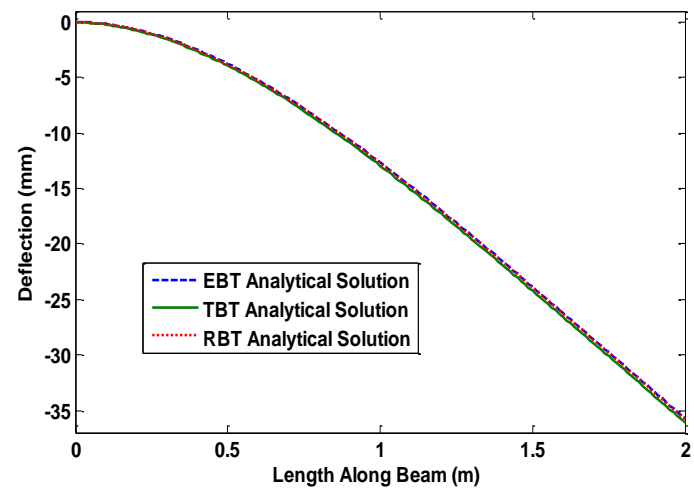

Fig. 17. Comparison of the analytical solutions in terms of deflections obtained by the EBT, TBT and RBT $(\mathrm{h} / \mathrm{L}=0.1)$

It is observed that the accuracy of the numerical results in terms of transverse deflection for TBT decrease with increasing $\mathrm{h} / \mathrm{L}$ ratio. The $\mathrm{h} / \mathrm{L}$ ratio is 0.1 in Fig. 17 . It is found that increasing of the $\mathrm{h} / \mathrm{L}$ ratio is decreasing the accuracy of the TBT in terms of transverse deflection as shown in Fig. 18.

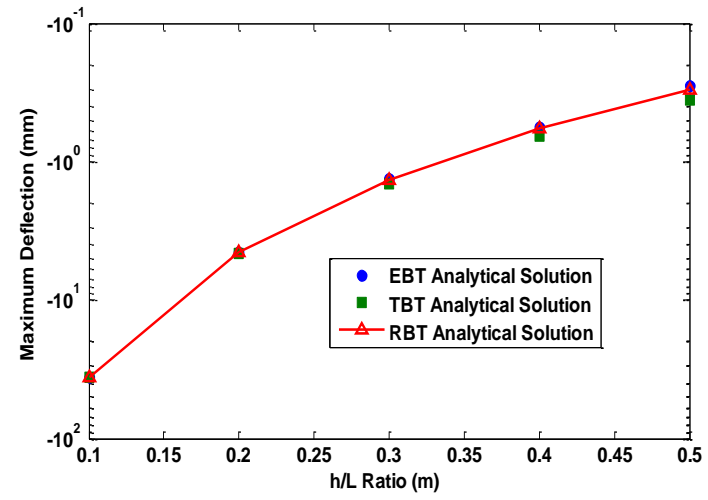

Fig. 18. Comparison of the analytical solutions in terms of maximum deflections obtained with varying $\mathrm{h} / \mathrm{L}$ ratio

\section{Conclusion}

The SSPH basis functions are employed to numerically solve the transverse deflections of the thick isotropic beams subjected to different sets of boundary conditions and uniformly distributed load by using strong formulation of the problem. The numerical calculations are performed by using different number of nodes uniformly distributed in the problem domain and by employing different beam theories which are the EBT, TBT and RBT. The performance of the SSPH method is investigated for the solution of the beam problems with the TBT and RBT for the first time. It is found that the SSPH method provides satisfactory results and 
convergence rate for the studied problems here. It is observed that the computed results of transverse deflections agree very well with the analytical solutions.

For the problems studied, it is found that the accuracy of the computed results based on the TBT is deteriorated by the aspect ratio $(\mathrm{h} / \mathrm{L})$. The accuracy of the transverse deflections computed by using analytical solution based on the TBT decreases with an increase in the aspect ratio of the beam.

It is observed that when the EBT formulation employed for the solution of the problems by using the SSPH method, the computed results in terms of the displacement are virtually indistinguishable from that for analytical solution and the solution obtained by the RBT formulation.

Based on the results of two numerical examples it is recommended that the SSPH method can be applied for solving linear beam problems by employing different shear deformation theories.

\section{Acknowledgements}

Author thanks to anonymous reviewers.

\section{References}

[1] Love, A.E.H., A Treatise on the Mathematical Theory of Elasticity, fourth ed. Dover Publications, New York, 1927.

[2] Timoshenko, S.P., Goodier, J.C., Theory of Elasticity, McGraw-Hill Co. Inc., New York, 1970.

[3] Wang, C.M., Reddy, J.N., Lee, K.H., Shear Deformable Beams and Plates Relations with Classical Solutions, Elsevier Science Ltd., Oxford 2000.

[4] Polizzotto, C., From the Euler-Bernoulli beam to the Timoshenko one through a sequence of Reddy-type shear deformable beam models of increasing order, European Journal of Mechanics A/Solids, 53, 62-74, 2015.

[5] Levinson, M., A new rectangular beam theory, Journal of Sound and Vibration, 74, 81-87, 1981.

[6] Bickford, W.B., A consistent higher order beam theory, Theor. Appl. Mech. 11, 137-150, 1982.

[7] Heyliger, P.R., Reddy, J.N., A higher order beam finite element for bending and vibration problems, Journal of Sound and Vibration, 126 (2), 309-326, 1988.

[8] Subramanian, P., Dynamic analysis of laminated composite beams using higher order theories and finite elements, Composite Structures, 73, 342-353, 2006.

[9] Reddy, J.N., Nonlocal theories for bending, buckling and vibration of beams, Int. J. Eng. Sci. 45, 288-307, 2007.

[10] Carrera, E., Giunta, G., Refined beam theories based on a unified formulation, Int. J. Appl. Mech. 2 (1), 117 $143,2010$.
[11] Giunta, G., Biscani, F., Bellouettar, S., Ferreira, A.J.M., Carrera, E., Free vibration analysis of composite beams via refined theories, Composites Part B, 44, 540-552, 2013.

[12] [12] Arya, H., A new zig-zag model for laminated composite beams: free vibration analysis, Journal of Sound and Vibration, 264, 485-490, 2003.

[13] Jun, L., Hongxing, H., Dynamic stiffness analysis of laminated composite beams using trigonometric shear deformation theory, Composite Structures, 89, 433-442, 2009.

[14] Kurama, M., Afaq, K.S., Mistou, S., Mechanical behavior of laminated composite beams by the new multi-layered laminated composite structures model with trigonometric shear stress continuity, Int. J. Solids Struct, 40, 1525-1546, 2003.

[15] Donning, B.M., Liu, W.K., Meshless methods for shear-deformable beams and plates, Computer Methods in Applied Mechanics and Engineering, 152, 47-71, 1998.

[16] Gu, Y.T., Liu, G.R., A local point interpolation method for static and dynamic analysis of thin beams, Computer Methods in Applied Mechanics and Engineering, 190, 42, 5515-5528, 2001.

[17] Ferreira, A.J.M., Roque, C.M.C., Martins, P.A.L.S., Radial basis functions and higher-order shear deformation theories in the analysis of laminated composite beams and plates, Composite Structures, 66, 287-293, 2004.

[18] Ferreira, A.J.M., Fasshauer, G.E., Computation of natural frequencies of shear deformable beams and plates by an RBF-pseudospectral method, Computer Methods in Applied Mechanics and Engineering, 196, 134-146, 2006.

[19] Moosavi, M.R., Delfanian, F., Khelil, A., The orthogonal meshless finite volume method for solving Euler-Bernoulli beam and thin plate problems, ThinWalled Structures, 49, 923-932, 2011.

[20] Roque, C.M.C., Figaldo, D.S., Ferreira, A.J.M., Reddy, J.N., A study of a microstructure-dependent composite laminated Timoshenko beam using a modified couple stress theory and a meshless method, Composite Structures, 96, 532-537, 2013.

[21] Lucy LB, A numerical approach to the testing of the fission hypothesis, Astronomical Journal, 82, 10131024, 1977.

[22] Chen JK, Beraun JE, Jin CJ, An improvement for tensile instability in smoothed particle hydrodynamics, Computational Mechanics, 23, 279-287, 1999.

[23] Chen JK, Beraun JE, Jin CJ, Completeness of corrective smoothed particlemethod for linear elastodynamics, Computational Mechanics, 24, 273-285, 1999. 
[24] Liu WK, Jun S, Zhang YF, Reproducing kernel particle methods, International Journal for Numerical Methods in Fluids, 20, 1081-1106, 1995.

[25] Liu WK, Jun S, Li S, Adee J, Belytschko T, Reproducing kernel particle methods for structural dynamics, International Journal for Numerical Methods in Engineering, 38, 1655-1679, 1995.

[26] Chen JS, Pan C,Wu CT, Liu WK, Reproducing kernel particle methods for large deformation analysis of nonlinear structures, Computer Methods in Applied Mechanics and Engineering, 139, 195-227, 1996.

[27] Zhang GM, Batra RC, Modified smoothed particle hydrodynamics method and its application to transient problems, Computational Mechanics, 34, 137-146, 2004.

[28] Batra RC, Zhang GM, Analysis of adiabatic shear bands in elasto-thermo- viscoplastic materials by modified smoothed particle hydrodynamics (MSPH) method, Journal of Computational Physics, 201, 172$190,2004$.

[29] Zhang GM, Batra RC, Wave propagation in functionally graded materials by modified smoothed particle hydrodynamics (MSPH) method, Journal of Computational Physics, 222, 374-390, 2007.

[30] Batra RC, Zhang GM, Search algorithm, and simulation of elastodynamic crack propagation by modified smoothed particle hydrodynamics (MSPH) method, Computational Mechanics, 40, 531-546, 2007.

[31] Zhang GM, Batra RC, Symmetric smoothed particle hydrodynamics (SSPH) method and its application to elastic problems, Computational Mechanics, 43, 321340, 2009.

[32] Batra RC, Zhang GM, SSPH basis functions for meshless methods, and comparison of solutions with strong and weak formulations, Computational Mechanics, 41, 527-545, 2008.

[33] Tsai CL, Guan YL, Batra RC, Ohanehi DC, Dillard JG, Nicoli E, Dillard DA, Comparison of the performance of SSPH and MLS basis functions for two-dimensional linear elastostatics problems including quasistatic crack propagation, Computational Mechanics, 51, 19-34, 2013.

[34] Tsai CL, Guan YL, Ohanehi DC, Dillard JG, Dillard DA, Batra RC, Analysis of cohesive failure in adhesively bonded joints with the SSPH meshless method, International Journal of Adhesion \& Adhesives, 51, 67-80, 2014.

[35] Karamanli A, Mugan A, Solutions of two-dimensional heat transfer problems by using symmetric smoothed particle hydrodynamics method, Journal of Applied and Computational Mathematics 1, 1-6, 2012.

[36] Karamanli A, Bending Deflection Analysis of a SemiTrailer Chassis by Using Symmetric Smoothed Particle Hydrodynamics, International Journal of Engineering Technologies, 1, 4, 134-140, 2015.

[37] Karamanli A, Mugan A, Strong from meshless implementation of Taylor series method, Applied Mathematics and Computation, 219, 9069-9080, 2013.

[38] Karamanli A, Different Implementation Approaches of the Strong Form Meshless Implementation of Taylor Series Method, International Journal of Engineering Technologies, Vol. 1, No:3, 2015.

[39] Kaewumpai, S., Luadsong, A., Two-field-variable meshless method based on moving kriging interpolation for solving simply supported thin plates under various loads. Journal of King Saud University - Science, 1018-3647, 2014.

[40] Yimnak, K., Luadsong, A., A local integral equation formulation based on moving kriging interpolation for solving coupled nonlinear reaction-diffusion equations. Advances in Mathematical Physics, 2014.

[41] Zhuang, X., Zhu, H., Augarde, C., The meshless Shepard and least squares (MSLS) method, Computational Mechanics, 53, 343-357, 2014.

[42] Fatahi, H., Nadjafi, JS, Shivanian, E, New spectral meshless radial point interpolation (SMRPI) method for the two-dimensional Fredholm integral equations on general domains with error analysis, Journal of Computational and Applied Mathematics, 264, 196209, 2016. 